\title{
An Improved Perturbation Pressure Closure for Eddy-Diffusivity Mass-Flux Schemes
}

\author{
Jia He ${ }^{1}$, Yair Cohen ${ }^{1}$, Ignacio Lopez-Gomez ${ }^{1}$, Anna Jaruga ${ }^{1}$, Tapio \\ Schneider ${ }^{1,2}$ \\ ${ }^{2}$ Jet Propulsion Laboratory, California Institute of Technology, Pasadena, California, USA.
}

\section{Key Points:}

- An analytical closure for the perturbation pressure in turbulence and convection parameterizations is derived.

- The closure combines the effects of virtual mass, momentum convergence, and pressure drag.

- The closure performs well in simulating a rising bubble and the diurnal cycle of deep convection.

Corresponding author: Tapio Schneider, tapio@caltech.edu 


\begin{abstract}
Convection parameterizations such as eddy-diffusivity mass-flux (EDMF) schemes require a consistent closure formulation for the perturbation pressure, which arises in the equations for vertical momentum and turbulence kinetic energy (TKE). Here we derive an expression for the perturbation pressure from approximate analytical solutions for $2 \mathrm{D}$ and 3D rising thermal bubbles. The new closure combines a modified pressure drag and virtual mass effects with a new momentum advection term. This momentum advection is an important source in the lower half of the thermal bubble and at cloud base levels in convective systems. It represents the essential physics of the perturbation pressure, that is, to ensure the 3D non-divergent properties of the flow. Moreover, the new formulation modifies the pressure drag to be inversely proportional to updraft depth. This is found to significantly improve simulations of the diurnal cycle of deep convection, without compromising simulations of shallow convection. It is thus a key step toward a unified scheme for a range of convective motions. By assuming that the pressure only redistributes TKE between plumes and the environment, rather than vertically, a closure for the velocity pressure-gradient correlation is obtained from the perturbation pressure closure. This novel pressure closure is implemented in an extended EDMF scheme and is shown to successfully simulate a rising bubble test case as well as shallow and deep convection cases in a single column model.
\end{abstract}

\title{
Plain Language Summary
}

Global climate models rely on subgrid-scale (SGS) parameterizations to represent heat and moisture transport by unresolved turbulent and convective motions. In this and two companion papers, the extended eddy-diffusivity mass-flux (EDMF) scheme is developed as a single unified scheme that represents all SGS turbulent and convective processes. This paper focuses on the closure for the perturbation pressure that ensures the non-divergence of the 3D mass flux. An analytical formulation for the pressure closure is derived by considering the dynamics of a buoyant bubble. The closure differs from commonly used formulations in two respects. First, it introduces an additional momentum advection term that contributes a momentum source at the bubble bottom and cloud base. Second, it improves the drag term and enables the EDMF to correctly reproduce the diurnal cycle of deep convection. Comparison with large-eddy simulations of moist convection and rising bubbles demonstrates the adequacy of the closure.

\section{Introduction}

Turbulent and convective motions play essential roles in the transport of energy and moisture in the climate system. Due to computational constraints, climate models use resolutions that are too coarse to resolve these motions and rely heavily on various parameterizations to represent their subgrid-scale (SGS) contribution to the resolved flow. Such parameterizations are one of the primary sources of model uncertainty in long term climate projections (Bony \& Dufresne, 2005; Bony et al., 2015; Brient \& Schneider, 2016; Caldwell et al., 2018; Ceppi et al., 2017; Murphy et al., 2004; Teixeira et al., 2011; Webb et al., 2013). Since advances in computational resources will not suffice to fully resolve turbulent and convective motions in the foreseeable future (Schneider et al., 2017), continuous efforts to reduce the biases and uncertainties from SGS parameterizations in climate models are required.

Conventionally, SGS processes such as boundary layer turbulence, shallow convection, and deep convection have been represented by separate parameterization schemes. This leads to a discontinuous representation of processes that lie on a physical continuum. It also results in a proliferation of correlated parameters (e.g., separate entrainment rates for shallow and deep convection), which complicates the calibration of climate models. Considerable efforts have been made to develop a unified parameteriza- 
tion that synthesizes the SGS turbulence and convection processes into one single scheme without artificial switches between different regimes (Lappen \& Randall, 2001a, 2001c, 2001b; Larson \& Golaz, 2005; Golaz et al., 2002b, 2002a; Soares et al., 2004; Siebesma et al., 2007; Park, 2014a, 2014b; Tan et al., 2018; Thuburn et al., 2018, 2019; Weller \& McIntyre, 2019; Cohen et al., 2020; Lopez-Gomez et al., 2020). A challenge in the development of such a unified scheme is closing the representation of various physical processes that emerge in the development of the scheme. In the case of mass-flux parameterizations, one of the key terms requiring closure is the perturbation pressure gradient, which is the focus of this work.

Perturbation pressure, defined as the departure of pressure from a reference profile in hydrostatic balance with a reference density, plays an important role in the development of convective systems (Holton, 1973; Schumann \& Moeng, 1991; Jeevanjee \& Romps, 2015, 2016; Morrison, 2016). It is an essential source/sink term for vertical momentum (Holton, 1973) and contributes to the redistribution of turbulence kinetic energy (TKE) (Heinze et al., 2015). It is typically diagnosed from a 3D Poisson equation in large-eddy simulations (LES), and its closure remains challenging for parameterization schemes (Holland \& Rasmusson, 1973; Morrison, 2016; Peters, 2016; Tarshish et al., 2018).

Some studies explicitly account for the perturbation pressure in modelling convective system, which usually requires a full set of equations that solves both vertical and horizontal motions (Holton, 1973; Lappen \& Randall, 2006; Morrison, 2016; Leger et al., 2019). Most parameterization schemes, however, do not explicitly solve for the pressure gradient term in their equation sets. Instead, the perturbation pressure gradient is formulated semi-empirically as a combination of various physical processes: a virtual mass effect that effectively reduces buoyancy, a momentum sink proportional to entrainment, and a drag term inversely proportional to the horizontal scale of the updraft (Simpson \& Wiggert, 1969; Siebesma et al., 2007; de Roode et al., 2012; Tan et al., 2018; Han \& Bretherton, 2019; Suselj et al., 2019).

The formulation as summarized in de Roode et al. (2012) represents a pure sink for the vertical momentum of convective systems. However, in an LES study, Jeevanjee and Romps (2015) decomposed the perturbation pressure into a buoyancy perturbation pressure and a dynamic perturbation pressure. They showed that the dynamic pressure is a significant momentum source at low levels of convective systems. Peters (2016) observed a similar positive momentum forcing from the dynamic perturbation pressure in a deep convective system. These results are in contradiction to the typical pressure closures that serve merely as a momentum sink. In this paper, we demonstrate that a vertical momentum source owing to the perturbation pressure gradient is important for capturing the dynamics of an idealized rising dry bubble, and this translates to shallow and deep convective systems.

We derive a novel closure for the perturbation pressure in the extended eddy-diffusivity mass-flux (EDMF) framework (Tan et al., 2018; Cohen et al., 2020). The closure explicitly recognizes the roles of the perturbation pressure as a vertical momentum source and sink and in TKE redistribution. The extended EDMF framework and its entrainment and detrainment closures are presented in Cohen et al. (2020), and the eddy diffusivity and mixing length closures are discussed in Lopez-Gomez et al. (2020). Together with the perturbation pressure closure, these closures make the extended EDMF a unified framework that successfully simulates a wide range of turbulent and convective regimes, from stable boundary layers to deep convection, without altering any of the equation components nor parameter values. Moreover, we show here that the extended EDMF scheme is also able to simulate individual convective bubbles, albeit with changes in the parameters and some additions to the formulation of the entrainment and detrainment closures. The need for these changes is discussed in the context of the general difference between convective updrafts and convective bubbles. 
Section 2 lays out the analytical derivation for the perturbation pressure in a $2 \mathrm{D}$ rising bubble, with the 3D counterpart given in Appendix B. Section 3 briefly reviews the extended EDMF framework and implements the perturbation pressure closure in it. Section 4 describes the setups of a dry bubble experiment and moist convective test cases in LES and a single column model (SCM). Simulation results are discussed in Section 5. Finally, Section 6 ends with the conclusions.

\section{Vertical Perturbation Pressure Gradient}

The momentum equation in the Boussinesq approximation is written as

$$
\frac{\partial \mathbf{v}}{\partial t}+\mathbf{v} \cdot \nabla \mathbf{v}=b \hat{\mathbf{k}}-\nabla\left(\frac{p^{\dagger}}{\rho_{h}}\right)+S_{\mathbf{v}}
$$

where $t$ is time, $\mathbf{v}=(u, v, w)$ is the $3 \mathrm{D}$ velocity vector, $\hat{\mathbf{k}}$ is the vertical unit vector, $\rho_{h}$ is a constant reference density, and $S_{\mathbf{v}}$ represents $3 \mathrm{D}$ momentum sources other than buoyancy and the pressure gradient force. The buoyancy is defined as

$$
b=-g \frac{\rho-\rho_{h}}{\rho},
$$

where $g$ is the gravitational acceleration. The perturbation pressure is defined as

$$
p^{\dagger}=p-p_{h},
$$

where $p_{h}(z)$ is the reference pressure profile in hydrostatic balance with the reference density $\rho_{h}$, i.e., $\hat{\mathbf{k}} \cdot \nabla p_{h}=-\rho_{h} g$.

\subsection{Pressure Poisson Equation}

With the Boussinesq approximation, replacing the density by the time-independent reference density in the continuity equation implies that the $3 \mathrm{D}$ velocity $\mathbf{v}$ is nondivergent. Therefore, taking the divergence of the momentum equation (1) and ignoring the source term $S_{\mathbf{v}}$ leads to a Poisson equation for the perturbation pressure

$$
\nabla^{2}\left(\frac{p^{\dagger}}{\rho_{h}}\right)=\frac{\partial b}{\partial z}-\nabla \cdot(\mathbf{v} \cdot \nabla \mathbf{v}) .
$$

To simplify notation, we define a pressure potential as

$$
P=\frac{p}{\rho_{h}} .
$$

In the remainder of this paper, we use the pressure potential $P$, which we generally refer to as "pressure" as it plays a similar role in the vertical momentum equation. We derive a closure for the gradient of the perturbation pressure potential, $\nabla P^{\dagger}$, with the dagger again denoting perturbations relative to the reference pressure potential.

It is common to decompose the perturbation pressure into the buoyancy perturbation pressure $\left(P_{\mathrm{b}}\right)$ and the dynamic perturbation pressure $\left(P_{\mathrm{d}}\right)$, associated with the two terms on the right-hand side of (2),

$$
\begin{aligned}
& \nabla^{2} P_{\mathrm{b}}=\frac{\partial b}{\partial z}, \\
& \nabla^{2} P_{\mathrm{d}}=-\nabla \cdot(\mathbf{v} \cdot \nabla \mathbf{v}) .
\end{aligned}
$$

In the derivations that follow, we consider for simplicity a 2D Cartesian geometry. An analogous derivation for an axisymmetric thermal bubble in cylindrical coordinates is given in Appendix B. In the 2D geometry, with $\mathbf{v}=(u, w)$ and $\nabla_{x, z}^{2}=\partial^{2} / \partial x^{2}+$ 

The coefficient $P_{0}$ is obtained by substituting this form for $P_{\mathrm{b}}$ into (8), leading to

$$
\nabla_{x, z}^{2} P_{\mathrm{b}}=P_{0} b_{A}\left[-\left(m^{2}+k^{2}\right) \cos (m z) \cos (k x)\right]=m b_{A} \cos (m z) \cos (k x) .
$$

This gives

$$
P_{0}=-\frac{m}{m^{2}+k^{2}}
$$

Therefore, the single normal mode solution for the buoyancy perturbation pressure is

$$
P_{\mathrm{b}}=-\frac{m}{m^{2}+k^{2}} b_{A} \cos (m z) \cos (k x),
$$

and the buoyancy perturbation pressure gradient needed in the vertical momentum equation is

$$
\frac{\partial P_{\mathrm{b}}}{\partial z}=\frac{m^{2}}{m^{2}+k^{2}} b_{A} \sin (m z) \cos (k x)=\frac{m^{2}}{m^{2}+k^{2}} b .
$$

\subsubsection{Dynamic Perturbation Pressure}

Similarly, we make the trigonometric ansatz (7) for the solution of the Poisson equation (6) for the dynamic perturbation pressure, leading to

$$
\begin{aligned}
\nabla_{x, z}^{2} P_{\mathrm{d}}=-2\left[\left(\frac{\partial w}{\partial z}\right)^{2}+\frac{\partial u}{\partial z} \frac{\partial w}{\partial x}\right] & =\frac{m^{2}}{2} w_{A}^{2} \cos (2 m z) \cos (2 k x) \\
& -\frac{m^{2}}{2} w_{A}^{2} \cos (2 k x)-\frac{3 m^{2}}{2} w_{A}^{2} \cos (2 m z)-\frac{m^{2} w_{A}^{2}}{2} .
\end{aligned}
$$

The first three terms on the right-hand side suggest a trigonometric structure for the dynamic perturbation pressure. The last term implies a drag-like quadratic term in the momentum equation (arising from a quadratic trigonometric identity). We write $P_{\mathrm{d}}$ in the same trigonometric structure as the right-hand side of (12), i.e.,

$$
P_{\mathrm{d}}=P_{1} w_{A}^{2} \cos (2 m z) \cos (2 k x)+P_{2} w_{A}^{2} \cos (2 k x)+P_{3} w_{A}^{2} \cos (2 m z)+F,
$$

where $P_{1}, P_{2}$, and $P_{3}$ are dimensionless coefficients that depend on the horizontal and vertical wavenumbers $k$ and $m$. The function $F$ satisfies $\nabla_{x, z}^{2} F=-m^{2} w_{A}^{2} / 2$ and represents the drag effects. The coefficients $P_{1}, P_{2}$, and $P_{3}$ are obtained by substituting this form for $P_{\mathrm{d}}$ into (12), leading to

$$
\begin{aligned}
\nabla_{x, z}^{2} P_{\mathrm{d}}=-4 P_{1}\left(m^{2}+k^{2}\right) w_{A}^{2} & \cos (2 m z) \cos (2 k x) \\
& -4 P_{2} k^{2} w_{A}^{2} \cos (2 k x)-4 P_{3} m^{2} w_{A}^{2} \cos (2 m z)+\nabla_{x, z}^{2} F .
\end{aligned}
$$

This gives

$$
\begin{aligned}
& P_{1}=-\frac{m^{2}}{8\left(m^{2}+k^{2}\right)}, \\
& P_{2}=\frac{m^{2}}{8 k^{2}} \\
& P_{3}=\frac{3}{8} \\
& \nabla_{x, z}^{2} F=-\frac{m^{2} w_{A}^{2}}{2} .
\end{aligned}
$$

is

Therefore, the single normal mode solution for the dynamic perturbation pressure

$$
P_{\mathrm{d}}=-\frac{m^{2}}{8\left(m^{2}+k^{2}\right)} w_{A}^{2} \cos (2 m z) \cos (2 k x)+\frac{m^{2}}{8 k^{2}} w_{A}^{2} \cos (2 k x)+\frac{3}{8} w_{A}^{2} \cos (2 m z)+F .
$$


The vertical gradient of the dynamic perturbation pressure is

$$
\frac{\partial P_{\mathrm{d}}}{\partial z}=-\frac{3 m}{4} w_{A}^{2} \sin (2 m z)+\frac{m^{3}}{4\left(m^{2}+k^{2}\right)} w_{A}^{2} \sin (2 m z) \cos (2 k x)+\frac{\partial F}{\partial z},
$$

where $F$ is the solution of $\nabla_{x, z}^{2} F=-m^{2} w_{A}^{2} / 2$.

This formulation of the perturbation pressure gradient was derived based on the normal-mode solution of an idealized thermal. In the following section, we briefly present the EDMF framework, which is based on conditional averages of the equations of motion, and we discuss how the normal-mode solution can form the basis of a closure for the perturbation pressure in the EDMF scheme.

\section{Perturbation Pressure in the Extended EDMF}

In the EDMF framework, a GCM grid box is divided into subdomains that consist of coherent updrafts/downdrafts and an isotropic environment. Following Cohen et al. (2020), the conditional average of a property $\phi$ in the $i$-th subdomain is denoted by $\bar{\phi}_{i}$, with $a_{i}$ as the area fraction occupied by the subdomain. The fluctuation around the subdomain average is denoted by $\phi_{i}^{\prime}=\phi-\bar{\phi}_{i}$. We use $i=0$ for the turbulent isotropic environment and $i \geq 1$ for coherent updrafts and downdrafts. Angle brackets $\langle\phi\rangle$ denote the grid-mean average of $\phi$, and $\phi^{*}=\phi-\langle\phi\rangle$ denotes the fluctuation around the grid mean. It is also convenient to define the difference between the subdomain average and the grid box average as $\bar{\phi}_{i}^{*}=\bar{\phi}_{i}-\langle\phi\rangle$. Finally, the grid box average is related to the subdomain average by the area-weighted average over all subdomains:

$$
\langle\phi\rangle=\sum_{i} a_{i} \bar{\phi}_{i}
$$

Using Reynolds averaging rules and this subdomain decomposition, SGS vertical fluxes are decomposed into the sum of subdomain-average components and components owing to fluctuations within the subdomains:

$$
\left\langle w^{*} \phi^{*}\right\rangle=\sum_{i} a_{i}\left(\bar{w}_{i}^{*} \bar{\phi}_{i}^{*}+\overline{w_{i}^{\prime} \phi_{i}^{\prime}}\right)
$$

The first term is represented by mass flux closures while the second term is taken to be nonzero only for the turbulent environment $(i=0)$ and is modeled as downgradient eddy diffusion, as in the name of the eddy-diffusivity mass-flux (EDMF) scheme. Accurate parameterization of this SGS vertical flux is the key goal of the EDMF scheme.

The full set of equations solved by the extended EDMF scheme is discussed in Cohen et al. (2020). For the purpose of understanding the role of perturbation pressure, here we briefly lay out the vertical momentum equation for updrafts/downdrafts, and the TKE equation for the environment, in which the perturbation pressure arises. 


\subsection{Updraft Vertical Velocity and Environmental TKE in the Extended EDMF}

The vertical momentum equation for the $i$-th subdomain is

$$
\begin{aligned}
\frac{\partial\left(\rho a_{i} \bar{w}_{i}\right)}{\partial t}+ & \nabla_{h} \cdot\left(\rho a_{i}\left\langle\mathbf{u}_{h}\right\rangle \bar{w}_{i}\right)+\frac{\partial\left(\rho a_{i} \bar{w}_{i} \bar{w}_{i}\right)}{\partial z}=\underbrace{\frac{\partial}{\partial z}\left(\rho a_{i} K_{w, i} \frac{\partial \bar{w}_{i}}{\partial z}\right)}_{\text {turbulent flux }} \\
& +\underbrace{\sum_{j \neq i}\left[\left(E_{i j}+\hat{E}_{i j}\right) \bar{w}_{j}-\left(\Delta_{i j}+\hat{E}_{i j}\right) \bar{w}_{i}\right]}_{\text {entrainment } / \text { detrainment }}+\underbrace{\rho a_{i} \bar{b}_{i}^{*}+\rho a_{i}\langle b\rangle}_{\text {buoyancy }} \\
& \underbrace{\left.-\rho a_{i} \frac{\partial P^{\dagger}}{\partial z}\right)_{i}^{*}-\rho a_{i} \frac{\partial\left\langle P^{\dagger}\right\rangle}{\partial z}}_{\text {perturbation pressure }},
\end{aligned}
$$

where $\mathbf{u}_{h}$ is the horizontal component of the velocity vector, whose subdomain value is taken to be equal to its grid-mean value. The exchange of mass is represented by dynamical entrainment, $E_{i j}$, dynamical detrainment, $\Delta_{i j}$, and turbulent entrainment, $\hat{E}_{i j}$; see Cohen et al. (2020) for details. Vertical turbulent fluxes are represented by the downgradient eddy diffusivity $K_{w, i}$ (Lopez-Gomez et al., 2020).

The subdomain buoyancy is defined as

$$
\bar{b}_{i}=-g \frac{\bar{\rho}_{i}-\rho_{h}}{\rho} .
$$

It is decomposed into a contribution from the grid-mean buoyancy

$$
\langle b\rangle=-g \frac{\rho-\rho_{h}}{\rho},
$$

and a departure from the grid mean

$$
\bar{b}_{i}^{*}=-g \frac{\bar{\rho}_{i}^{*}-\rho_{h}}{\rho} .
$$

Similarly, the perturbation pressure gradient is decomposed into a grid-mean component and a departure from the grid mean, i.e.,

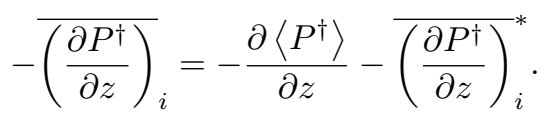

In the GCM setting, the grid-mean buoyancy $\langle b\rangle$ and perturbation pressure gradient $-\partial\left\langle P^{\dagger}\right\rangle / \partial z$ are provided by the dynamical core; in the SCM setting, they are balanced as in Eq. (47) in Cohen et al. (2020). The subdomain buoyancy relative to the grid mean, $\bar{b}_{i}^{*}$, is computed from the density using a nonlinear saturation adjustment; see the appendix in Pressel et al. (2015). Here we develop a closure scheme for the subdomain perturbation pressure, $-{\overline{\left(\partial P^{\dagger} / \partial z\right)_{i}}}^{*}$. 
The subdomain TKE is defined as $\bar{e}_{i}=0.5\left(\overline{u_{i}^{\prime 2}}+\overline{v_{i}^{\prime 2}}+\overline{w_{i}^{\prime 2}}\right)$, and the environmental TKE equation is

$$
\begin{aligned}
& \frac{\partial\left(\rho a_{0} \bar{e}_{0}\right)}{\partial t}+\nabla_{h} \cdot\left(\rho a_{0}\left\langle\mathbf{u}_{h}\right\rangle \bar{e}_{0}\right)+\frac{\partial\left(\rho a_{0} \bar{w}_{0} \bar{e}_{0}\right)}{\partial z}= \\
& \underbrace{\frac{\partial}{\partial z}\left(\rho a_{0} K_{m, 0} \frac{\partial \bar{e}_{0}}{\partial z}\right)}_{\text {turbulent transport }}+\underbrace{\rho a_{0} K_{m, 0}\left[\left(\frac{\partial\langle u\rangle}{\partial z}\right)^{2}+\left(\frac{\partial\langle v\rangle}{\partial z}\right)^{2}+\left(\frac{\partial \bar{w}_{0}}{\partial z}\right)^{2}\right]}_{\text {shear production }} \\
& +\sum_{i>0}(\underbrace{-\hat{E}_{0 i} \bar{e}_{0}}_{\text {turb. entrainment }}+\underbrace{\bar{w}_{0}^{*} \hat{E}_{0 i}\left(\bar{w}_{0}-\bar{w}_{i}\right)}_{\text {turb. entrainment production }}) \\
& +\sum_{i>0}(\underbrace{-\Delta_{0 i} \bar{e}_{0}}_{\text {dyn. detrainment }}+\frac{1}{2} \underbrace{E_{0 i}\left(\bar{w}_{0}-\bar{w}_{i}\right)\left(\bar{w}_{0}-\bar{w}_{i}\right)}_{\text {dyn. entrainment production }}) \\
& +\underbrace{\rho a_{0} \overline{w_{0}^{\prime} b_{0}^{\prime}}}_{\text {buoyancy production }}-\underbrace{\rho a_{0} \overline{\left[u_{0}^{\prime}\left(\frac{\partial P^{\dagger}}{\partial x}\right)_{0}^{\prime}\right.}+\overline{v_{0}^{\prime}\left(\frac{\partial P^{\dagger}}{\partial y}\right)_{0}^{\prime}}+\overline{w_{0}^{\prime}\left(\frac{\partial P^{\dagger}}{\partial z}\right)_{0}^{\prime}}}_{\text {pressure work }}-\underbrace{\rho a_{0} \bar{D}_{e, 0}}_{\text {dissipation }},
\end{aligned}
$$

with TKE dissipation denoted by $\bar{D}_{e, 0}$. Closure schemes for the shear production, entrainment and detrainment, turbulent transport, buoyancy production, and dissipation are discussed in Cohen et al. (2020) and Lopez-Gomez et al. (2020).

The pressure work in the environment can be computed using

$$
-\rho a_{0}\left[\overline{w_{0}^{\prime}\left(\frac{\partial P^{\dagger}}{\partial z}\right)_{0}^{\prime}}+\overline{u_{0}^{\prime}\left(\frac{\partial P^{\dagger}}{\partial x}\right)_{0}^{\prime}}+\overline{v_{0}^{\prime}\left(\frac{\partial P^{\dagger}}{\partial y}\right)_{0}^{\prime}}=\sum_{i \geq 1} \rho a_{i}\left(\bar{w}_{i}^{*}-\bar{w}_{0}^{*}\right) \overline{\left(\frac{\partial P^{\dagger}}{\partial z}\right)_{i}^{*}},\right.
$$

once the perturbation pressure gradient is closed for the momentum equations in the updrafts and downdrafts. This equation assumes that subdomain covariance within updrafts and downdrafts are negligible, a general assuption in EDMF schemes. A derivation of this relation is provided in Appendix A, given the assumption that pressure perturbations only redistribute TKE between subdomains and do no work on the grid mean (Tan et al., 2018). It is noteworthy that (22) is different from how the pressure work term is closed in higher-order turbulence schemes (e.g., Bretherton and Park (2009)), which usually combines the pressure work into the turbulent TKE transport and parameterizes the entire transport term diffusively.

\subsection{Implementation of Perturbation Pressure Closure in the Extended EDMF Scheme}

Equation (11) provides the buoyancy perturbation pressure forcing under the single normal mode assumption. It applies to the vertical momentum equation for any point within a rising thermal. To use this within the EDMF scheme, we apply a conditional average to evaluate the integrated effect of the pressure gradient forcing on a rising thermal. Let the conditional average over the thermal be denoted as

$$
\bar{\phi}_{\mathrm{thm}}(z)=\frac{1}{2 R} \int_{-R}^{R} \phi(x, z) \mathrm{d} x .
$$

Then, the vertical gradient of the buoyancy perturbation pressure (11) at height $z$ is

$$
\overline{\left(\frac{\partial P_{\mathrm{b}}}{\partial z}\right)_{\mathrm{thm}}}=\frac{1}{2 R} \int_{-R}^{R} \frac{m^{2}}{m^{2}+k^{2}} b \mathrm{~d} x=\frac{m^{2}}{m^{2}+k^{2}} \bar{b}_{\mathrm{thm}}=\frac{1}{1+\left(\frac{H}{2 R}\right)^{2}} \bar{b}_{\mathrm{thm}} .
$$

This is proportional to the buoyancy of the thermal and hence modifies the buoyancy term in the vertical momentum equation. This virtual mass effect makes the effective 



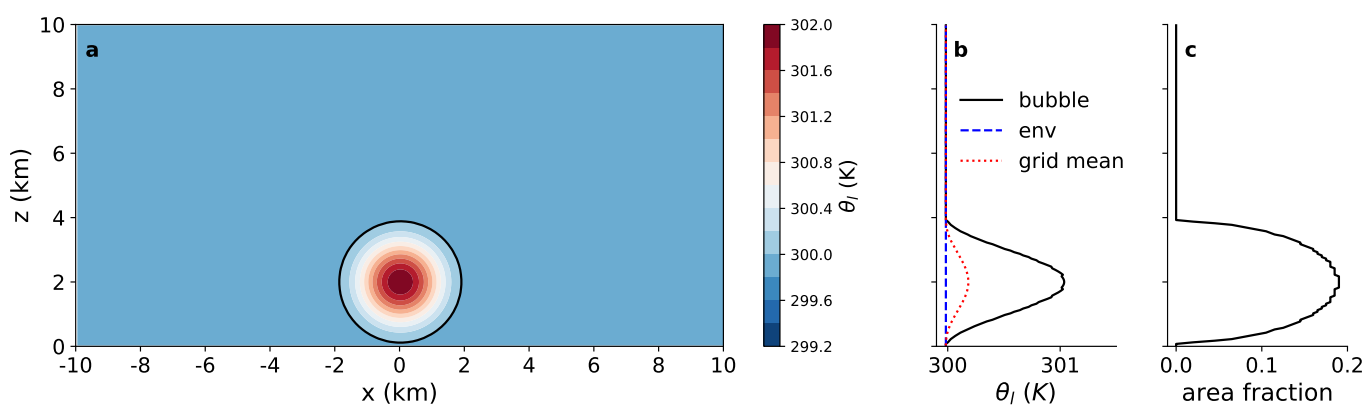

Figure 1. Initial profiles of the rising bubble experiments in LES. a: Contours of $\theta_{l}$ with intervals of $0.2 \mathrm{~K}$. The black contour is at $300 \mathrm{~K}$ and it outlines the edge of the initial bubble that is used for the conditional average computation. b: Initial vertical profiles of $\theta_{l}$ conditionally averaged over the bubble (black solid line) and the environment (blue dashed line), as well as the grid-mean $\theta_{l}$ (red dotted line). c: Initial profile of the bubble area fraction.

environmental TKE equation. The performance of the EDMF scheme is compared with LES. The LES experiments are performed with PyCLES (Pressel et al., 2015), an anelastic atmospheric LES code with entropy and total water specific humidity as prognostic variables, designed to simulate boundary layer turbulence and convection. We examine the structure of a dry rising bubble following the benchmark test in Bryan and Fritsch (2002), but also compare it to individually selected thermals in observationally motivated test cases of moist convection.

\subsection{D Rising Bubble}

\subsubsection{LES Setup}

The rising bubble experiment runs on a $2 \mathrm{D}$ domain of $10 \mathrm{~km}$ in height and $20 \mathrm{~km}$ in width. The initial liquid water potential temperature $\left(\theta_{l}\right)$ distribution over the domain is

$$
\theta_{l}(x, z)= \begin{cases}300 \mathrm{~K}+2 \mathrm{~K} \cos ^{2}(0.5 \pi L(x, z)), & \text { if } L<1, \\ 300 \mathrm{~K}, & \text { if } L \geq 1 .\end{cases}
$$

where

$$
L=\sqrt{\left(\frac{x-x_{c}}{x_{r}}\right)^{2}+\left(\frac{z-z_{c}}{z_{r}}\right)^{2}}
$$

represents the normalized distance from the point $(x, z)$ to the bubble center $x_{c}=10 \mathrm{~km}$ and $z_{c}=2 \mathrm{~km}$, and $x_{r}=z_{r}=2 \mathrm{~km}$ represent the initial radius of the bubble. This initial $\theta_{l}$ distribution is unstable near $x_{c}$ and stable far from it (Figure 1a). The thermal bubble contains the strongest warm anomaly in the bubble center, which decays toward the edge of the bubble. The liquid water potential temperature $\theta_{l}$ is homogeneous outside the bubble, creating an almost neutral environment. Both the environment and the bubble are initially at rest. The buoyancy force associated with the perturbed $\theta_{l}$ field provides the initial momentum source for the bubble to rise.

\subsubsection{SCM Setup}

The SCM simulation is initialized by taking the conditional average over the bubble from the LES initial setup. The buoyant bubble is identified by the $300 \mathrm{~K} \theta_{l}$-contour (black contour in Figure 1a). The initial updraft area fraction is computed as the ratio of the horizontal extent of the bubble over the horizontal LES domain size as shown in 
Figure 1c. Initial $\theta_{l}$ for the updraft is computed as the conditional average of $\theta_{l}$ within the perturbed area, shown in Figure 1b. Also shown are the grid-mean and environmental profiles of initial $\theta_{l}$. This initial $\theta_{l}$ profile introduces a positively buoyant bubble into a negatively buoyant environment. The updraft velocity is initialized as zero throughout the column, consistent with the resting initial state in LES. No external forcing is applied along the simulation.

As discussed in Cohen et al. (2020), subdomain horizontal velocities are assumed equal to the grid-mean horizontal velocity and changes in area fraction due to horizontal mass exchange are attributed to dynamical entrainment and detrainment. A rising bubble results in a large mass and momentum convergence at the bubble bottom and divergence at the top (Sánchez et al., 1989). This requires an additional divergence term in addition to the dynamical entrainment and detrainment. Therefore, the entrainment and detrainment rates for the bubble test case are modified as

$$
\begin{gathered}
E_{i j}=\tilde{E}_{i j}+\rho c_{\text {div }} \max \left(\frac{\mathrm{d}\left(a_{i} w_{i}\right)}{\mathrm{d} z}, 0\right), \\
\Delta_{i j}=\tilde{\Delta}_{i j}+\rho c_{\text {div }} \max \left(-\frac{\mathrm{d}\left(a_{i} w_{i}\right)}{\mathrm{d} z}, 0\right),
\end{gathered}
$$

where $c_{\text {div }}=0.4$ is a scaling coefficient, and $\tilde{E}_{i j}$ and $\tilde{\Delta}_{i j}$ are the entrainment and detrainment rates proposed by Cohen et al. (2020). The second term is an addition for the bubble test case only; it has been implemented in an EDMF scheme for simulating oceanic convection (Giordani et al., 2020) and a multi-fluid framework for the thermal bubble (Weller et al., 2020). The bubble test case is an initial value problem that is different from the typical boundary value problems for turbulence and convection that a SGS model needs to simulate in a climate model, and hence the introduction of these additional terms, not present in Cohen et al. (2020) and Lopez-Gomez et al. (2020), may be justified.

\subsection{Moist Convection}

Atmospheric convective systems consist of large numbers of thermal bubbles (Morrison, 2017), which can be identified by their dynamical and thermodynamic properties (e.g., Romps and Charn (2015)). A convective parameterization attempts to represent the statistical mean of these bubbles. Here we test the EDMF framework with the proposed pressure closure against a shallow convection case from the Barbados Oceanographic and Meteorological Experiment (BOMEX, Holland and Rasmusson (1973)) and a deep convection case from the Tropical Rainfall Measurement Mission Large-scale Biosphere-Atmosphere experiment (TRMM-LBA, Grabowski et al. (2006)). The LES and SCM simulations for BOMEX and TRMM-LBA follow the experimental setups described in Cohen et al. (2020). The pressure closure takes $(29)$ with $\left(\alpha_{b}, \alpha_{a}, \alpha_{d}\right)=(0.12,0.1,10.0)$ as the scaling constants. The closures for entrainment and detrainment are given in equations (31) and (32) in Cohen et al. (2020), that is, without the divergence term as described above for the bubble case. The eddy diffusivity and mixing length in the environment are closed as in Lopez-Gomez et al. (2020). At the same time, the results in these companion papers rely on the pressure closure derived in this work.

Following Couvreux et al. (2010), a passive tracer is added for the LES simulation. A 3D mask that identifies updrafts in moist convection is obtained based on criteria on the vertical velocity, tracer concentration, and liquid water specific humidity as described in Cohen et al. (2020). We compute the bulk properties of convective plumes by taking the conditional average over the updraft mask. Against these bulk properties, we compare the performance of the updraft profiles in the SCM simulations.

To investigate the structure of individual thermal bubbles in moist convection, we manually identify bubbles from the 3D field outputs for the last simulation timestep. The 

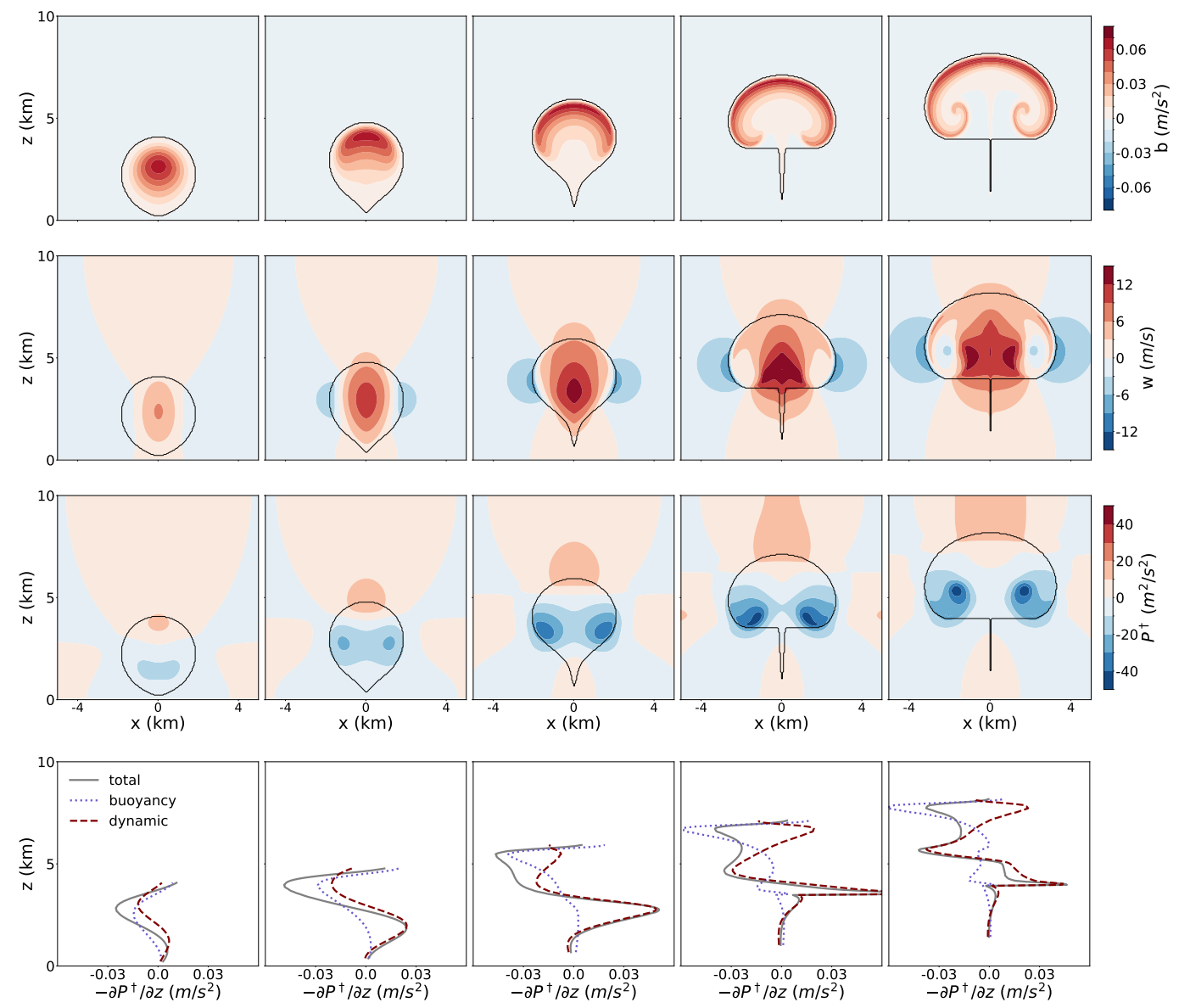

Figure 2. Snapshots of the rising bubble in 200-second intervals. The black contour in each contour plot traces the bubble boundary. From left to right are bubbles at 200, 400, 600, 800, and 1000 seconds into the simulation. The first 3 rows from top to bottom are buoyancy, vertical velocity, and perturbation pressure potential $P^{\dagger}$. The bottom row shows conditional averages of the perturbation pressure gradient force $-\partial_{z} P^{\dagger}$ and its decomposition into the buoyancy and dynamic components.

thermal bubbles are identified by sweeping over the 3D fields. We search from the cloudtop level down to the cloud-base level for BOMEX. For TRMM-LBA, we search for convective thermals that grow above $3 \mathrm{~km}$. To exclude negatively buoyant structures, which can occur near cloud top when convective overshoots occur, from being identified as updrafts, we remove regions of negative buoyancy from the tracer-based updraft identification. In the end, we identify 13 convective thermals from BOMEX and 8 from TRMMLBA for a composite study.

A composite of the thermal bubbles is created to illustrate their robust structures in $w$, buoyancy, $P^{\dagger}$ and $-\partial_{z} P^{\dagger}$. First, for each bubble, an azimuthal average is computed around the vertical axis that goes through the location of the maximum $w$ in the bubble. Then, the composite is created by aligning the $2 \mathrm{D}$ azimuthal averages of each bubble by their locations of maximum $w$. 

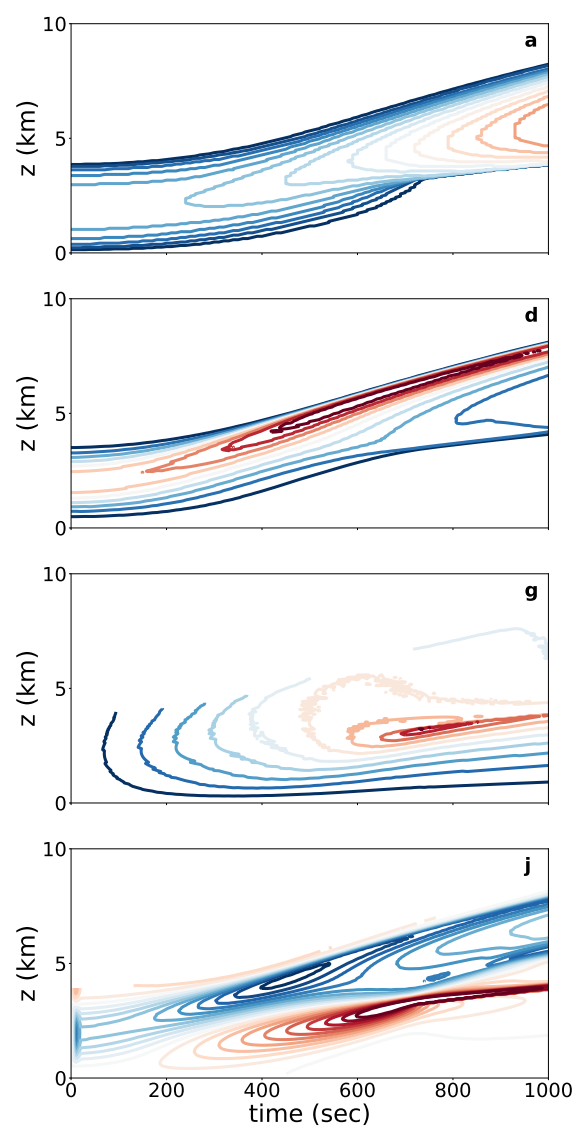
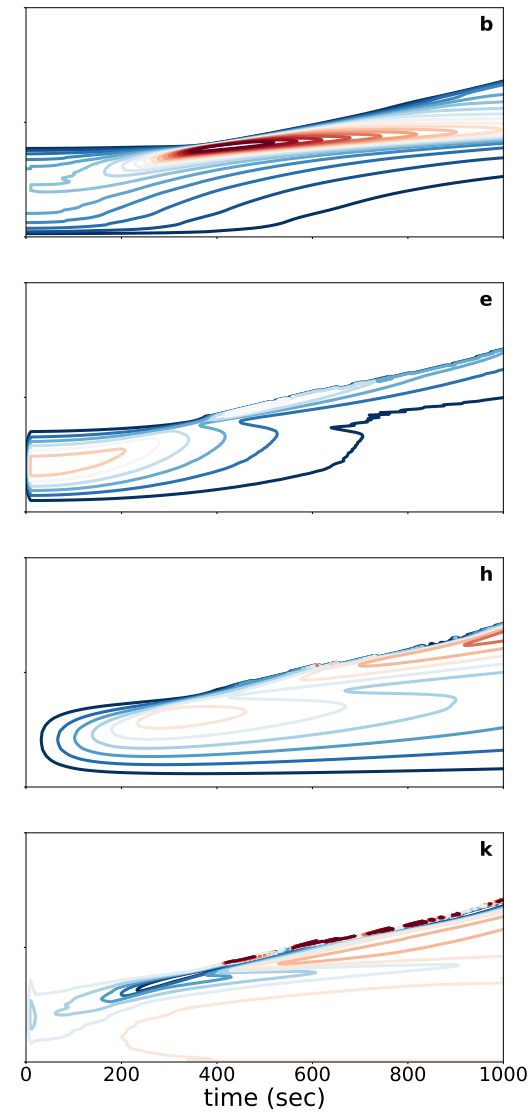
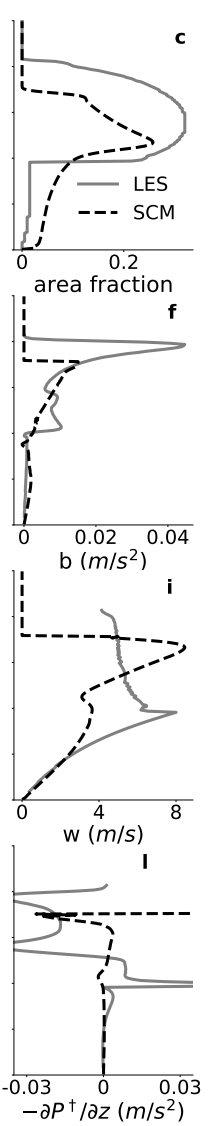

Figure 3. Comparison of bubble structures between LES and SCM simulations. a: Time evolution of the bubble area fraction in LES. Contours from blue to red represent $[0.06,0.08$, ..., 0.30]. b: Time evolution of the bubble area fraction in SCM. Contours from blue to red represent $[0.06,0.08, \ldots, 0.40]$. c: Vertical profiles of area fraction for the last time step of the LES (solid) and SCM (dashed) simulations. d: Time evolution of the bubble buoyancy in LES. Contours from blue to red represent $[0.005,0.010, \ldots, 0.045] \mathrm{m} \mathrm{s}^{-2}$. e: Time evolution of the bubble buoyancy in SCM. Contours from blue to red represent $[0.005,0.010, \ldots, 0.030] \mathrm{m} \mathrm{s}^{-2}$. f: As in $\mathbf{c}$ but for buoyancy. g: Time evolution of the bubble vertical velocity in LES. Contours from blue to red represent $[1,2, \ldots, 9] \mathrm{m} \mathrm{s}^{-1}$. h: Time evolution of the bubble vertical velocity in SCM. Contours from blue to red represent $[1,2, \ldots, 8] \mathrm{m} \mathrm{s}^{-1}$. i: As in c but for vertical velocity. j: Time evolution of the bubble $-\partial_{z} P^{\dagger}$ in LES. Contours from blue to red represent $[-0.05$, $-0.045, \ldots, 0.045] \mathrm{m} \mathrm{s}^{-2}$. k: Time evolution of the bubble $-\partial_{z} P^{\dagger}$ in LES. Contours from blue to red represent $[-0.01,-0.008, \ldots, 0.01] \mathrm{m} \mathrm{s}^{-2}$. $\mathbf{l}$ : As in $\mathbf{c}$ but for $-\partial_{z} P^{\dagger}$. 


\section{Results}

\subsection{D Rising Bubble}

Snapshots of the bubble structure from LES are shown in Figure 2. Similar to Figure 1a, the bubble is outlined by black contours with zero buoyancy. Given this initial buoyancy distribution, the upward vertical velocity builds up quickly inside the bubble, while compensating downdrafts are established and closely wrap the rising bubble. This is a robust structure in convective elements and captures well the vertical fluxes of heat and moisture in convective systems ( $\mathrm{Gu}$ et al., 2020). Meanwhile, a negative perturbation pressure is established below the maximum buoyancy level, while a positive perturbation pressure is established above it. As the buoyancy center is pushed upward as the bubble rises, the zero perturbation pressure contour line moves toward the bubble top, and negative $P^{\dagger}$ dominates the majority of the bubble. A peak in negative $P^{\dagger}$ develops at the center of the bubble, which results in a momentum source from the perturbation pressure gradient below this level and a momentum sink above it. The bottom panels in Figure 2 show the conditional average of the pressure gradient force and its decomposition into buoyancy and dynamic components. At the bottom and mid-levels of the bubble, $-\partial_{z} P_{\mathrm{d}}^{\dagger}$ dominates and is a momentum source at the lower part of the bubble and a sink at its top. The buoyancy component, $-\partial_{z} P_{\mathrm{b}}^{\dagger}$, contributes primarily as a sink offsetting the buoyancy field.

During the early stages of the simulation (i.e., before $600 \mathrm{~s}$ ), the $2 \mathrm{D}$ structure of the buoyancy and velocity fields resemble the trigonometric structure assumed in (7). Therefore, the single normal mode assumption is a reasonable simplification. The perturbation pressure exhibits a dumbbell structure in the lower bubble, which indicates the dynamic perturbation pressure associated with velocity plays an essential role at these levels. Toward the end of the simulation, when the bubble deforms, the underlying assumption of a single normal mode structure becomes problematic as the strong buoyancy is pushed to the bubble's top while the maximum vertical velocity falls into the lower half of the bubble. However, a close investigation of the moist convective cases in the next subsection shows that individual bubbles in the convective system resemble the rising bubble structures during the early stages, which validates the single normal mode assumption made in the derivation.

The SCM with the extended EDMF parameterization and the pressure closure simulates well the time evolution of a rising thermal bubble, with greater success at early stages, as shown in Figure 3. The time evolution shows a rising bubble that for the most part detaches from the surface and maintain a coherent buoyancy anomaly. As the bubble rises, the maximum buoyancy level in the SCM simulation shifts from the bubble's center to its top, in agreement with the LES results. The area fraction shows a slightly sharper gradient at the top of the bubble at around 400 seconds. The SCM also roughly captures the vertical velocity evolution in the LES. At the last time step of the simulations, the SCM produces a top-heavy vertical velocity profile with too high velocities at the bubble's top and too low velocities at its bottom. The area fraction profile in the SCM struggles to maintain the bubble structure and develops a spike at about $4.5 \mathrm{~km}$. In spite of these differences, the SCM produces a bubble that has many key features of the LES bubble, such as the area fraction, vertical extent, and the top and base heights (see Figure 3c).

Overall, this demonstrates the capability of the EDMF framework with the pressure closure to simulate a rising bubble.

\subsection{Moist Convection}

Thermal bubbles identified from the BOMEX and TRMM-LBA LES experiments demonstrate similar structures to the early stage of the rising thermal bubble experiment. 

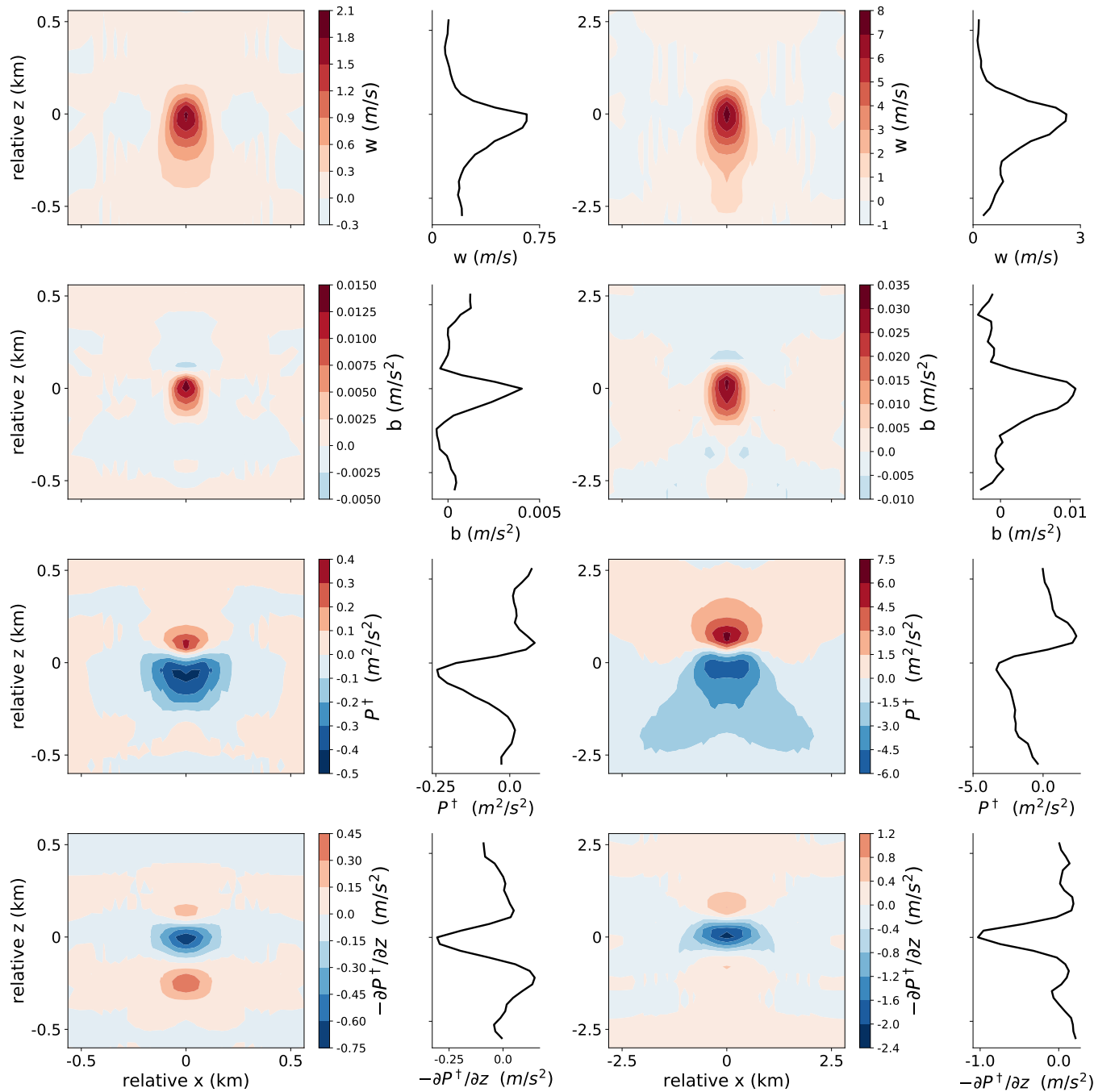

Figure 4. Average structures of bubble composites identified from LES simulations for BOMEX (left two columns) and TRMM-LBA (right two columns). Contour plots represent the azimuthally averaged structures of $w$, buoyancy, $P^{\dagger}$, and $-\partial_{z} P^{\dagger}$. The $x$ and $y$ axis in the contour plots represent the relative distances from the location of maximum vertical velocity. Column 2 (BOMEX) and 4 (TRMM-LBA) show the horizontal average of the bubble properties. Rows from top to bottom show vertical velocity, buoyancy, $P^{\dagger}$, and $-\partial_{z} P^{\dagger}$. 

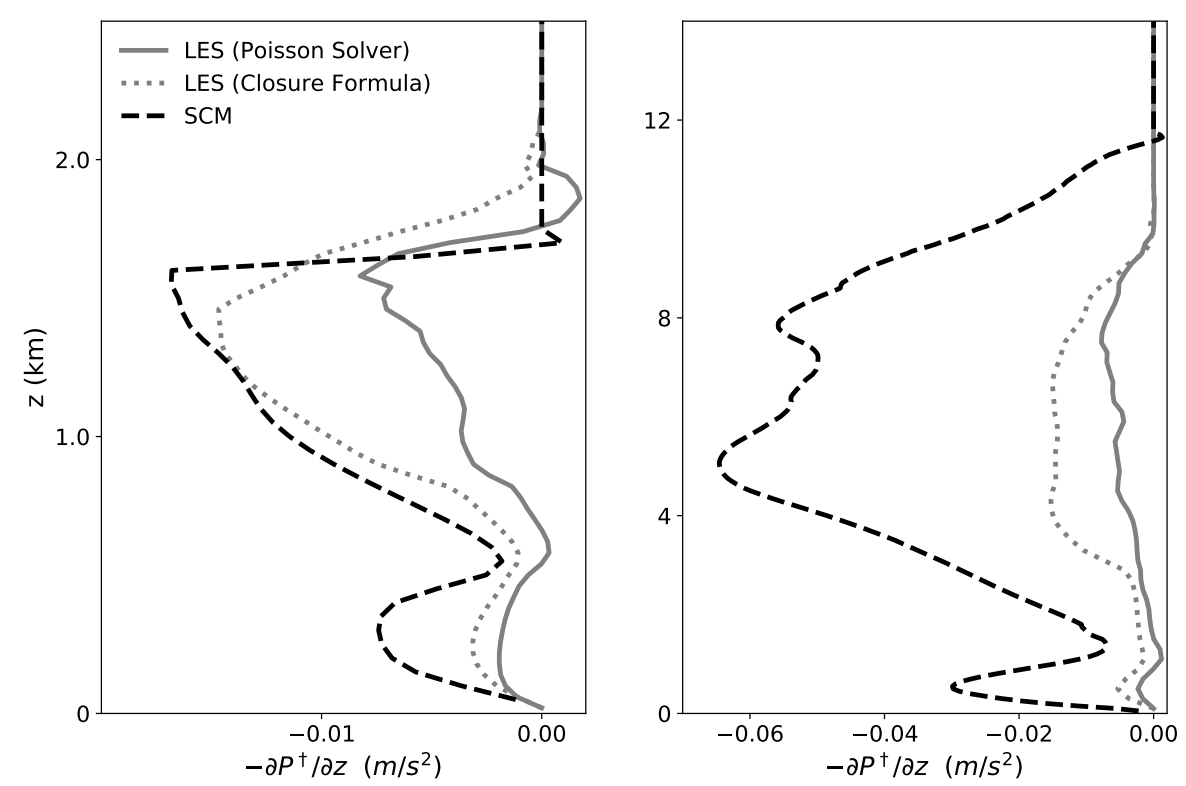

Figure 5. Comparison of $-\partial_{z} P^{\dagger}$ between LES and SCM for BOMEX (left) and TRMM-LBA (right). Pressure in the LES is shown in the grey solid line; pressure diagnosed from LES using (29) is shown in the grey dotted line; pressure from SCM is shown in the black dashed line.

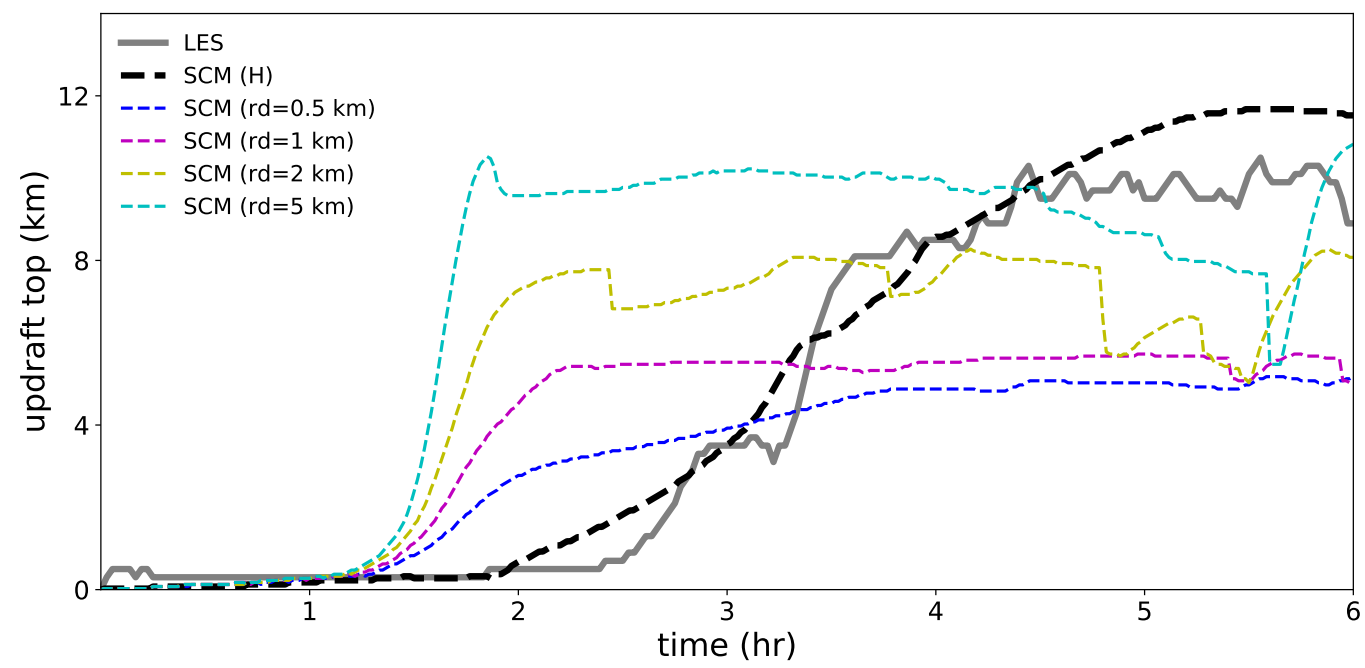

Figure 6. Comparisons of the TRMM-LBA cloud top evolution between LES (grey solid line) and SCM simulations with different pressure drag closures. SCM results with updraft height as length scale, as given by (17), are shown in the black dashed line. SCM results with characteristic plume radius as length scale in the pressure drag, as given by (34), are shown in the colored dashed lines for different values of $r_{d}$. 
Figure 4 shows the vertical velocity, buoyancy, perturbation pressure potential, and $-\partial_{z} P^{\dagger}$ profiles for a composite of bubbles selected in the BOMEX and TRMM-LBA test cases. The buoyancy profiles resemble an early-stage bubble. The perturbation pressure fields show the clear pattern of low pressure at the middle and lower levels of the bubble and high pressure at the top. The dumbbell structure characterizing the later stages of the rising bubble experiment does not show up in the composite (averaged) fields in Figure 4; however, they do show up if one looks into the individual bubbles instead of the composite. They are smoothed out when averaged over several bubbles with various horizontal and vertical extents. Averaged over many bubbles, the momentum source from $-\partial_{z} P^{\dagger}$ at bottom of the bubble and the sink at the top remain similar to the structure found in the rising bubble experiment. The resemblance between the composite of bubbles from moist convection and the idealized bubble experiment justifies the implementation of the single normal mode solution in the EDMF framework.

Cohen et al. (2020) demonstrate the capability of the EDMF framework to represent dynamic and thermodynamic properties within the updrafts, as well as their first, second, and third moments. Here we focus on the performance of the pressure closure (29) in the BOMEX and TRMM-LBA cases through comparison between the LES and SCM simulations (Figure 5). Comparing the pressure closure profiles in the SCM (dashed) with that diagnosed from (29) in LES (dotted), the SCM pressure closures represent the LES vertical profile quite well for the BOMEX case. For the TRMM-LBA case, the pressure gradient profile in SCM represents a much larger momentum sink compared to the LES. This is primarily due to an overestimation of the buoyancy variations in the SCM, which leads to a larger sink in the buoyancy perturbation pressure component. The compensation between these two in the end gives a reasonable representation of the vertical velocity profile, as shown in Cohen et al. (2020). Comparing the pressure gradient profiles as diagnosed from (29) (dotted) with that solved from the LES Poisson solver (solid), the former is about twice the magnitude of the latter. This is due to a considerable drag effect $\left(\alpha_{d}=10.0\right)$. The large drag effect is needed as a stabilization requirement (Weller \& McIntyre, 2019). Unlike for the rising bubble, the pressure gradient force for the bulk updrafts in BOMEX and TRMM-LBA acts primarily as a momentum sink.

An advantage of the current pressure closure manifests when examining the diurnal cycle of deep convection in SCM simulations and LES. Simulating the diurnal cycle is a major challenge for many parameterization schemes (Dai \& Trenberth, 2004; Holtslag et al., 2013). Here we show the effect of the length scale used in the denominator of the pressure drag on the timing of deep convection. When using a fixed scale, e.g., the updraft radius (Simpson \& Wiggert, 1969; Tan et al., 2018), a trade-off arises between improving the onset timing of convection and improving the cloud top height. In Tan et al. (2018), the pressure drag term in the $i$-th subdomain is written as

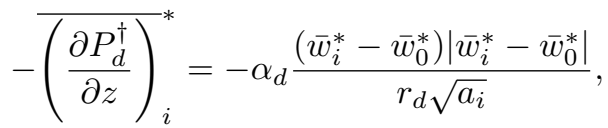

where $r_{d}=500 \mathrm{~m}$ is the typical distance between neighboring plumes in shallow convection; thus, $r_{d} \sqrt{a_{i}}$ gives a characteristic plume radius. However, our derivation indicates that the drag effect scales with the vertical scale of the convective system. Figure 6 compares the evolution of updraft tops in SCM and LES. The SCM simulations have fixed coefficients $\alpha_{b}=0.12$ and $\alpha_{a}=0.1$. We compare the drag term in (29) with that in (34) as in Tan et al. (2018). The value $r_{d}=500 \mathrm{~m}$ reproduces shallow convection as in Tan et al. (2018), but it leads to too early onset and too low updraft tops for the deep convective case. A simple increase in $r_{d}$ results in a universal decrease in the drag contribution and produces higher updraft tops. However, this does not solve the problem of the onset timing. Physically, convection in the TRMM-LBA case requires a large drag in the early stages, so that the convection is not initiated too early, and a gradually decreasing drag later, so that convection can grow high enough. The height of the updraft top, which arises in the normal mode derivation above, is therefore a natural scale. 


is

In the axisymmetric cylindrical coordinate system, the mass continuity equation

$$
\frac{\partial(u r)}{\partial r}+\frac{\partial(w r)}{\partial z}=0
$$
is

The pressure Poisson equation in the axisymmetric cylindrical coordinate system

$$
\nabla_{r, z}^{2} P^{\dagger}=\frac{\partial b}{\partial z}-\left[\left(\frac{\partial u}{\partial r}\right)^{2}+\left(\frac{u}{r}\right)^{2}+\left(\frac{\partial w}{\partial z}\right)^{2}\right]-2 \frac{\partial u}{\partial z} \frac{\partial w}{\partial r}
$$

Using the mass continuity equation, it simplifies to

$$
\nabla_{r, z}^{2} P^{\dagger}=\frac{\partial b}{\partial z}-2\left[\left(\frac{\partial w}{\partial z}\right)^{2}+\frac{\partial u}{\partial z} \frac{\partial w}{\partial r}-\frac{u}{r} \frac{\partial u}{\partial r}\right]
$$

where

$$
\nabla_{r, z}^{2}=\frac{1}{r} \frac{\partial}{\partial r} r \frac{\partial}{\partial r}+\frac{\partial^{2}}{\partial z^{2}}
$$

Similarly, the perturbation pressure potential is decomposed into the sum of buoyancy and dynamic perturbation pressure, i.e., $P^{\dagger}=P_{\mathrm{b}}+P_{\mathrm{d}}$ such that

$$
\begin{aligned}
& \nabla_{r, z}^{2} P_{\mathrm{b}}=\frac{\partial b}{\partial z} \\
& \nabla_{r, z}^{2} P_{\mathrm{d}}=-2\left[\left(\frac{\partial w}{\partial z}\right)^{2}+\frac{\partial u}{\partial z} \frac{\partial w}{\partial r}-\frac{u}{r} \frac{\partial u}{\partial r}\right]
\end{aligned}
$$


For a rising thermal, a trigonometric basis is used for the vertical wave structure, as for the $2 \mathrm{D}$ derivation, while Bessel functions of the first kind $J_{\alpha}(\cdot)$ are used for the horizontal structure, to exploit eigenfunctions of the Laplacian operator. That is

$$
\begin{aligned}
w & =w_{A} \sin (m z) J_{0}(k r), \\
b & =b_{A} \sin (m z) J_{0}(k r), \\
u & =u_{A} \cos (m z) J_{1}(k r),
\end{aligned}
$$

where $m=\pi H^{-1}$ is the vertical wavenumber, and $k=2.4 R^{-1}$ ensures $k R$ is the first zero of the Bessel function, $J_{0}(k R)=0$. Then, the mass continuity gives

$$
k u_{A}+m w_{A}=0
$$

which is essential in simplifying the following derivation.

\section{B1 Buoyancy Perturbation Pressure}

The buoyancy perturbation pressure satisfies

$$
\nabla_{r, z}^{2} P_{\mathrm{b}}=m b_{A} \cos (m z) J_{0}(k r) .
$$

With the eigenfunction ansatz, this can be solved to give

$$
P_{\mathrm{b}}=-\frac{m}{m^{2}+k^{2}} b_{A} \cos (m z) J_{0}(k r),
$$

which gives the buoyancy perturbation pressure gradient as

$$
\frac{\partial P_{\mathrm{b}}}{\partial z}=\frac{m^{2}}{m^{2}+k^{2}} b_{A} \sin (m z) J_{0}(k r)=\frac{m^{2}}{m^{2}+k^{2}} b .
$$

Therefore, the conditional average of the buoyancy perturbation pressure over the rising thermal is

$$
\overline{\left(\frac{\partial P_{\mathrm{b}}}{\partial z}\right)_{\mathrm{thm}}}=\frac{1}{R} \int_{0}^{R} \frac{m^{2}}{m^{2}+k^{2}} b r \mathrm{~d} r=\frac{m^{2}}{m^{2}+k^{2}} \bar{b}_{\mathrm{thm}}=\frac{1}{1+\left(\frac{2.4}{\pi} \frac{H}{2 R}\right)^{2}} \bar{b}_{\mathrm{thm}} .
$$

\section{B2 Dynamic Perturbation Pressure}

In cylindrical coordinates, the dynamic pressure includes a third term arising from the curvature of the coordinate system. The expansion of the dynamic perturbation pressure is done separately for each of the three terms as follows:

$$
\begin{gathered}
\left(\frac{\partial w}{\partial z}\right)^{2}=\left(m w_{A} \cos (m z) J_{0}(k r)\right)^{2} \\
=\frac{m^{2}}{2} w_{A}^{2}(1+\cos (2 m z)) J_{0}^{2}(k r) \\
\frac{\partial u}{\partial z} \frac{\partial w}{\partial r}=\left[-m u_{A} \sin (m z) J_{1}(k r)\right]\left[w_{A} \sin (m z)\left(-k J_{1}(k r)\right)\right] \\
=m k u_{A} w_{A} \sin ^{2} m z J_{1}^{2}(k r), \\
-\frac{u}{r} \frac{\partial u}{\partial r}=-\left[\frac{u_{A}}{r} \cos (m z) J_{1}(k r)\right]\left[u_{A} \cos (m z)\left(k J_{0}(k r)-\frac{J_{1}(k r)}{r}\right)\right] \\
=-\frac{u_{A}^{2}}{2}(1+\cos (2 m z))\left(\frac{k J_{0}(k r) J_{1}(k r)}{r}-\frac{J_{1}^{2}(k r)}{r^{2}}\right)
\end{gathered}
$$


Using these expansions, the Poisson equation for dynamic perturbation pressure is written as

$$
\begin{aligned}
\nabla_{r, z}^{2} P_{\mathrm{d}}= & -m^{2} w_{A}^{2}(1+\cos (2 m z)) J_{0}^{2}(k r)+m^{2} w_{A}^{2}(1-\cos (2 m z)) J_{1}^{2}(k r) \\
& +u_{A}^{2}(1+\cos (2 m z))\left(\frac{k J_{0}(k r) J_{1}(k r)}{r}-\frac{J_{1}^{2}(k r)}{r^{2}}\right) \\
= & -m^{2} w_{A}^{2}\left[J_{0}^{2}(k r)-J_{1}^{2}(k r)\right]-m^{2} w_{A}^{2} \cos (2 m z)\left[J_{0}^{2}(k r)+J_{1}^{2}(k r)\right] \\
& +\underbrace{u_{A}^{2}(1+\cos (2 m z))\left[\frac{k J_{0}(k r) J_{1}(k r)}{r}-\frac{J_{1}^{2}(k r)}{r^{2}}\right]}_{J_{r}} \\
= & -m^{2} w_{A}^{2} \frac{1+3 J_{0}(2 k r)}{4}-m^{2} w_{A}^{2} \cos (2 m z) \frac{3+J_{0}(2 k r)}{4}+J_{r} \\
= & -\frac{m^{2}}{4} w_{A}^{2} \cos (2 m z) J_{0}(2 k r)-\frac{3 m^{2}}{4} w_{A}^{2} \cos (2 m z)-\frac{3 m^{2}}{4} w_{A}^{2} J_{0}(2 k r) \\
- & \frac{m^{2}}{4} w_{A}^{2}+J_{r} .
\end{aligned}
$$

Similar to (12), the first three terms in (B14) have a wave structure while the fourth term, $-m^{2} w_{A}^{2} / 4$, represents a drag. Therefore, we make the ansatz that $P_{\mathrm{d}}$ has a structure consisting of a combination of $\cos (2 m z)$ and $J_{0}(2 k r)$, plus a drag function, $F$. The $J_{r}$ term in (B14) arises from $\mathrm{d}^{2} J_{1}^{2}(k r) / \mathrm{d} r^{2}$. Since $\mathrm{d}^{2} J_{1}^{2}(k r) / \mathrm{d} r^{2}$ is a function of $J_{0}(2 k r)$, we can simply assume an extra $J_{1}^{2}(k r)$ term in $P_{\mathrm{d}}$ first, and later combine it with the $J_{0}(2 k r)$ in the original assumption. Therefore, we write $P_{\mathrm{d}}=P_{\mathrm{d} 1}+P_{\mathrm{d} 2}$ where $P_{\mathrm{d} 1}$ corresponds to $J_{r}$ and $P_{\mathrm{d} 2}$ is the remaining combination of $\cos (2 m z)$ and $J_{0}(2 k r)$. By comparing $J_{r}$ and $\mathrm{d}^{2} J_{1}^{2}(k r) / \mathrm{d} r^{2}$, we have

$$
\begin{aligned}
& P_{\mathrm{d} 1}=-\frac{m^{2} w_{0}^{2}}{4 k^{2}}(1+\cos (2 m z)) J_{1}^{2}(k r) \\
& P_{\mathrm{d} 2}=P_{1} \cos (2 m z) J_{0}(2 k r)+P_{2} J_{0}(2 k r)+P_{3} \cos (2 m z)+F,
\end{aligned}
$$

where $F$ represents the drag, as in the $2 \mathrm{D}$ derivation. $P_{1}, P_{2}$, and $P_{3}$ are the magnitude of the three modes.

The Poisson equation for dynamic perturbation pressure becomes

$$
\begin{aligned}
\nabla_{r, z}^{2} P_{\mathrm{d}}= & \nabla_{r, z}^{2} P_{\mathrm{d} 1}+\nabla_{r, z}^{2} P_{\mathrm{d} 2} \\
= & -\left[4\left(m^{2}+k^{2}\right) P_{1}+\frac{3 m^{2}}{8} w_{A}^{2}+\frac{m^{2}}{4} u_{A}^{2}\right] \cos (2 m z) J_{0}(2 k r) \\
& -\left(4 k^{2} P_{2}+\frac{3 m^{2}}{8} w_{A}^{2}\right) J_{0}(2 k r)-\left(4 m^{2} P_{3}+\frac{m^{2}}{8} w_{A}^{2}-\frac{m^{2}}{4} u_{A}^{2}\right) \cos (2 m z) \\
& +\nabla_{r, z}^{2} F-\frac{3 m^{2}}{8} w_{A}^{2}+J_{r} .
\end{aligned}
$$

Comparison with the corresponding terms in (B14) leads to

$$
\begin{aligned}
P_{1} & =-\frac{m^{2}\left(k^{2}+2 m^{2}\right)}{32 k^{2}\left(k^{2}+m^{2}\right)} w_{A}^{2}, \\
P_{2} & =\frac{3 m^{2}}{32 k^{2}} w_{A}^{2}, \\
P_{3} & =\frac{5 k^{2}+2 m^{2}}{32 k^{2}} w_{A}^{2} .
\end{aligned}
$$

The dynamic perturbation pressure becomes

$$
\begin{aligned}
P_{\mathrm{d}} & =P_{\mathrm{d} 1}+P_{\mathrm{d} 2}=-\frac{m^{2} w_{0}^{2}}{4 k^{2}}(1+\cos (2 m z)) J_{1}^{2}(k r)+P_{1} \cos (2 m z) J_{0}(2 k r)+P_{2} J_{0}(2 k r)+P_{3} \cos (2 m z)+F \\
& =\frac{m^{2}}{32\left(m^{2}+k^{2}\right)} w_{A}^{2} \cos (2 m z) J_{0}(2 k r)+\frac{5 m^{2}}{32 k^{2}} w_{A}^{2} J_{0}(2 k r)+\frac{5}{32} w_{A}^{2} \cos (2 m z)+F-\frac{m^{2}}{16 k^{2}} w_{A}^{2}
\end{aligned}
$$


and the dynamic perturbation pressure gradient is

$$
\frac{\partial P_{\mathrm{d}}}{\partial z}=-\frac{m^{3}}{16\left(m^{2}+k^{2}\right)} w_{A}^{2} \sin (2 m z) J_{0}(2 k r)-\frac{5 m}{16} w_{A}^{2} \sin (2 m z)+\frac{\partial F}{\partial z} .
$$

The conditional average of a scalar over the rising thermal is computed as

$$
\bar{\phi}_{\mathrm{thm}}=\frac{1}{\pi R^{2}} \int_{0}^{2 \pi} \mathrm{d} \theta \int_{0}^{R} \phi r \mathrm{~d} r=\frac{2}{R^{2}} \int_{0}^{R} \phi r \mathrm{~d} r .
$$

Therefore, the dynamic perturbation pressure averaged over the rising thermal is

$$
\begin{aligned}
\overline{\left(\frac{\partial P_{\mathrm{d}}}{\partial z}\right)_{\mathrm{thm}}=} & \underbrace{-\frac{2}{R^{2}} \int_{0}^{R} \frac{m^{3}}{16\left(m^{2}+k^{2}\right)} w_{A}^{2} \sin (2 m z) J_{0}(2 k r) r \mathrm{~d} r}_{A} . \\
& \underbrace{-\frac{2}{R^{2}} \int_{0}^{R} \frac{5 m}{16} w_{A}^{2} \sin (2 m z) r \mathrm{~d} r}_{B}+\underbrace{\frac{2}{R^{2}} \int_{0}^{R} \frac{\partial F}{\partial z} r \mathrm{~d} r}_{C} .
\end{aligned}
$$

The first term on the right-hand side integrates to

$$
\begin{aligned}
A & =-\frac{2}{R^{2}} \int_{0}^{R} \frac{m^{3}}{16\left(m^{2}+k^{2}\right)} w_{A}^{2} \sin (2 m z) J_{0}(2 k r) r \mathrm{~d} r \\
& =-\frac{m^{3}}{8\left(m^{2}+k^{2}\right)} \frac{w_{A}^{2}}{R^{2}} \sin (2 m z) \int_{0}^{R} J_{0}(2 k r) r \mathrm{~d} r \\
& =-\frac{m^{3}}{8\left(m^{2}+k^{2}\right)} \frac{w_{A}^{2}}{R^{2}} \sin (2 m z) \frac{R}{2 k} J_{1}(2 k R) \\
& =-\frac{m^{3}}{16\left(m^{2}+k^{2}\right)} \frac{w_{A}^{2}}{k R} \sin (2 m z) J_{1}(2 k R) \\
& =-\frac{m^{2}}{32\left(m^{2}+k^{2}\right)} \frac{k R J_{1}(2 k R)}{J_{1}^{2}(k R)} \bar{w}_{\mathrm{thm}} \frac{\mathrm{d} \bar{w}_{\mathrm{thm}}}{\mathrm{d} z},
\end{aligned}
$$

where $k R \approx 2.4$ corresponds to the zero $J_{0}(k r)=0$. The second term integrates to

$$
\begin{aligned}
B & =-\frac{2}{R^{2}} \int_{0}^{R} \frac{5 m}{16} w_{A}^{2} \sin (2 m z) r \mathrm{~d} r \\
& =-\frac{5 m}{16} w_{A}^{2} \sin (2 m z) \\
& =-\frac{5 k^{2} R^{2}}{32 J_{1}^{2}(k R)} \bar{w}_{\mathrm{thm}} \frac{\mathrm{d} \bar{w}_{\mathrm{thm}}}{\mathrm{d} z}
\end{aligned}
$$

Therefore. the sum of the first two terms on the right-hand side, $A+B$, gives the advective term. In the axisymmetric system, the advective term is also dependent on the shape of the thermal, i.e., $k / m$ which is different from the $2 \mathrm{D}$ Cartesian bubble. This is mainly due to the fact that $\cos (2 k x)$ integrates to zero from $-R$ to $R$, as in term $B$ of (25), while $J_{0}(2 k x)$ does not integrate to zero, as in term $A$ of (B20).

Term $C$ is the drag term and can be justified in the same way as in the $2 \mathrm{D}$ derivation.

Therefore, the perturbation pressure gradient force in a 3D bubble consists of the same three contributions as derived from the $2 \mathrm{D}$ bubble: the virtual mass term, a momentum advection term, and a drag term.

\section{Acknowledgments}

This research was made possible by the generosity of Eric and Wendy Schmidt by recommendation of the Schmidt Futures program, by Earthrise Alliance, Mountain Philanthropies, the Paul G. Allen Family Foundation, and the National Science Foundation (NSF, 
award AGS-1835860). We would like to thank the Resnick Sustainability Institute at Caltech for fellowship support. Parts of the research were carried out at the Jet Propulsion Laboratory, California Institute of Technology, under a contract with the National Aeronautics and Space Administration and funded through the internal Research and Technology Development program. The PyCLES code used to generate LES results is available at climate-dynamics.org/software/\#pycles. The SCM code is available at https:// doi.org/10.5281/zenodo.4291143.

(c) 2020. California Institute of Technology. Government sponsorship acknowledged.

\section{References}

Bony, S., \& Dufresne, J.-L. (2005). Marine boundary layer clouds at the heart of tropical cloud feedback uncertainties in climate models. Geophysical Research Letters, 32(20). doi: https://doi.org/10.1029/2005GL023851

Bony, S., Stevens, B., Frierson, D. M., Jakob, C., Kageyama, M., Pincus, R., ... others (2015). Clouds, circulation and climate sensitivity. Nature Geoscience, 8(4), 261-268. doi: 0.1038/ngeo2398

Bretherton, C. S., \& Park, S. (2009). A new moist turbulence parameterization in the community atmosphere model. Journal of Climate, 22(12), 3422-3448. doi: https://doi.org/10.1175/2008JCLI2556.1

Brient, F., \& Schneider, T. (2016). Constraints on climate sensitivity from spacebased measurements of low-cloud reflection. Journal of Climate, 29(16), 58215835. doi: https://doi.org/10.1175/JCLI-D-15-0897.1

Bryan, G. H., \& Fritsch, J. M. (2002). A benchmark simulation for moist nonhydrostatic numerical models. Monthly Weather Review, 130(12), 2917-2928. doi: https://doi.org/10.1175/1520-0493(2002)130〈2917:ABSFMN $>2.0 . C O ; 2$

Caldwell, P. M., Zelinka, M. D., \& Klein, S. A. (2018). Evaluating emergent constraints on equilibrium climate sensitivity. Journal of Climate, 31(10), 39213942. doi: 10.1175/JCLI-D-17-0631.1

Ceppi, P., Brient, F., Zelinka, M. D., \& Hartmann, D. L. ～(2017). Cloud feedback mechanisms and their representation in global climate models. Wiley Interdisciplinary Reviews: Climate Change, 8(4), e465. doi: 10.1002/wcc.465

Cohen, Y., Lopez-Gomez, I., Jaruga, A., He, J., Kaul, C. M., \& Schneider, T. (2020). Unified entrainment and detrainment closures for extended eddydiffusivity mass-flux schemes. Journal of Advances in Modeling Earth Systems, 12(9), e2020MS002162. doi: 10.1029/2020MS002162

Couvreux, F., Hourdin, F., \& Rio, C. (2010). Resolved versus parametrized boundary-layer plumes. part i: A parametrization-oriented conditional sampling in large-eddy simulations. Boundary-layer meteorology, 134 (3), 441-458. doi: $10.1007 / \mathrm{s} 10546-009-9456-5$

Dai, A., \& Trenberth, K. E. (2004). The diurnal cycle and its depiction in the community climate system model. Journal of Climate, 17(5), 930-951. doi: https://doi.org/10.1175/1520-0442(2004)017〈0930:TDCAID $>2.0 . C O ; 2$

Davies-Jones, R. (2003). An expression for effective buoyancy in surroundings with horizontal density gradients. Journal of the atmospheric sciences, $60(23), 2922-2925$. doi: https://doi.org/10.1175/1520-0469(2003)060<2922: AEFEBI $>2.0 . \mathrm{CO} ; 2$

de Roode, S. R., Siebesma, A. P., Jonker, H. J. J., \& de Voogd, Y. (2012). Parameterization of the vertical velocity equation for shallow cumulus clouds. Monthly Weather Review, 140(8), 2424-2436. doi: 10.1175/MWR-D-11-00277.1

Doswell III, C. A., \& Markowski, P. M. (2004). Is buoyancy a relative quantity? Monthly weather review, 132(4), 853-863. doi: https://doi.org/10.1175/1520 -0493(2004)132〈0853:IBARQ $\rangle 2.0 . C O ; 2$ 
Giordani, H., Bourdallé-Badie, R., \& Madec, G. (2020). An eddy-diffusivity massflux parameterization for modelling oceanic convection. Journal of Advances in Modeling Earth Systems, e2020MS002078. doi: https://doi.org/10.1029/ 2020MS002078

Golaz, J.-C., Larson, V. E., \& Cotton, W. R. (2002a). A pdf-based model for boundary layer clouds. part ii: Model results. Journal of the Atmospheric Sciences, $59(24), 3552-3571$. doi: https://doi.org/10.1175/1520-0469(2002)059/3552: APBMFB 2 2.0.CO;2

Golaz, J.-C., Larson, V. E., \& Cotton, W. R. (2002b). A pdf-based model for boundary layer clouds. part i: Method and model description. Journal of the Atmospheric Sciences, 59(24), 3540-3551. doi: https://doi.org/10.1175/

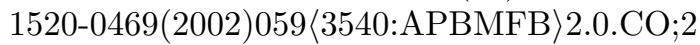

Grabowski, W., Bechtold, P., Cheng, A., Forbes, R., Halliwell, C., Khairoutdinov, M., ... et al. (2006). Daytime convective development over land: A model intercomparison based on lba observations. Quarterly Journal of the Royal Meteorological Society, 132(615), 317-344. doi: doi.org/10.1256/qj.04.147

Gu, J.-F., Plant, R. S., Holloway, C. E., Jones, T. R., Stirling, A., Clark, P. A., ... Webb, T. L. (2020). Evaluation of the bulk mass flux formulation using largeeddy simulations. Journal of the Atmospheric Sciences, 77(6), 2115-2137. doi: https://doi.org/10.1175/JAS-D-19-0224.1

Han, J., \& Bretherton, C. S. (2019). Tke-based moist eddy-diffusivity mass-flux (edmf) parameterization for vertical turbulent mixing. Weather and Forecasting , 34 (4), 869-886. doi: 10.1175/WAF-D-18-0146.1

Heinze, R., Mironov, D., \& Raasch, S. (2015). Second-moment budgets in cloud topped boundary layers: A large-eddy simulation study. Journal of Advances in Modeling Earth Systems, 7(2), 510-536. doi: 10.1002/2014MS000376

Holland, J. Z., \& Rasmusson, E. M. (1973). Measurements of the atmospheric mass, energy, and momentum budgets over a 500-kilometer square of tropical ocean. Monthly Weather Review, 101(1), 44-57. doi: 10.1175/ 1520-0493(1973)101<0044:MOTAME $\rangle$ 2.3.CO;2

Holton, J. R. (1973). A one-dimensional cumulus model including pressure perturbations. Monthly Weather Review, $101(3), 201-205$. doi: 10.1175/1520 -0493(1973)101〈0201:AOCMIP〉2.3.CO;2

Holtslag, A., Svensson, G., Baas, P., Basu, S., Beare, B., Beljaars, A., ... others (2013). Stable atmospheric boundary layers and diurnal cycles: challenges for weather and climate models. Bulletin of the American Meteorological Society, 94 (11), 1691-1706. doi: https://doi.org/10.1175/BAMS-D-11-00187.1

Jeevanjee, N., \& Romps, D. M. (2015). Effective buoyancy, inertial pressure, and the mechanical generation of boundary layer mass flux by cold pools. Journal of the Atmospheric Sciences, 72 (8), 3199-3213. doi: 10.1175/JAS-D-14-0349.1

Jeevanjee, N., \& Romps, D. M. (2016). Effective buoyancy at the surface and aloft. Quarterly Journal of the Royal Meteorological Society, 142(695), 811-820. doi: https://doi.org/10.1002/qj.2683

Lappen, C.-L., \& Randall, D. A. (2001a). Toward a unified parameterization of the boundary layer and moist convection. part i: A new type of mass-

flux model. Journal of the Atmospheric Sciences, 58(15), 2021-2036. doi: https://doi.org/10.1175/1520-0469(2001)058〈2021:TAUPOT $\rangle 2.0 . C O ; 2$

Lappen, C.-L., \& Randall, D. A. (2001b). Toward a unified parameterization of the boundary layer and moist convection. part iii: Simulations of clear and cloudy convection. Journal of the Atmospheric Sciences, 58(15), 2052-2072. doi: https://doi.org/10.1175/1520-0469(2001)058〈2052:TAUPOT $\rangle 2.0 . C O ; 2$

Lappen, C.-L., \& Randall, D. A. (2001c). Toward a unified parameterization of the boundary layer and moist convection. part ii: Lateral mass exchanges and subplume-scale fluxes. Journal of the Atmospheric Sciences, 58(15), 2037-2051. doi: https://doi.org/10.1175/1520-0469(2001)058〈2037:TAUPOT〉2.0.CO;2 

RG003i003p00387

Simpson, J., \& Wiggert, V. (1969). Models of precipitating cumulus towers. Mon. Wea. Rev, 97(7), 471-489. doi: https://doi.org/10.1175/ 1520-0493(1969)097〈0471:MOPCT $\rangle 2.3 . \mathrm{CO} ; 2$

Soares, P. M. M., Miranda, P. M. A., Siebesma, A. P., \& Teixeira, J. (2004). An eddy-diffusivity/mass-flux parametrization for dry and shallow cumulus convection. Quarterly Journal of the Royal Meteorological Society, 130(604), 3365-3383. doi: 10.1256/qj.03.223

Suselj, K., Kurowski, M. J., \& Teixeira, J. (2019). On the factors controlling the development of shallow convection in eddy-diffusivity/mass-flux models. Journal of the Atmospheric Sciences, 76 (2), 433-456. doi: 10.1175/JAS-D-18-0121.1

Tan, Z., Kaul, C. M., Pressel, K. G., Cohen, Y., Schneider, T., \& Teixeira, J. (2018). An extended eddy-diffusivity mass-flux scheme for unified representation of subgrid-scale turbulence and convection. Journal of Advances in Modeling Earth Systems, 10(3), 770-800. doi: 10.1002/2017MS001162

Tarshish, N., Jeevanjee, N., \& Lecoanet, D. (2018). Buoyant motion of a turbulent thermal. Journal of the Atmospheric Sciences, 75(9), 3233-3244. doi: 10.1175/ JAS-D-17-0371.1

Teixeira, J., Cardoso, S., Bonazzola, M., Cole, J., DelGenio, A., DeMott, C., ... others (2011). Tropical and subtropical cloud transitions in weather and climate prediction models: The gcss/wgne pacific cross-section intercomparison (gpci). Journal of Climate, 24(20), 5223-5256. doi: 10.1175/2011JCLI3672.1

Thuburn, J., Efstathiou, G. A., \& Beare, R. J. (2019). A two-fluid single-column model of the dry, shear-free, convective boundary layer. Quarterly Journal of the Royal Meteorological Society, 145(721), 1535-1550. doi: 10.1002/qj.3510

Thuburn, J., Weller, H., Vallis, G. K., Beare, R. J., \& Whitall, M. (2018). A framework for convection and boundary layer parameterization derived from conditional filtering. Journal of the Atmospheric Sciences, 75(3), 965-981. doi: 10.1175/JAS-D-17-0130.1

Webb, M. J., Lambert, F. H., \& Gregory, J. M. (2013). Origins of differences in climate sensitivity, forcing and feedback in climate models. Climate Dynamics, 40(3-4), 677-707. doi: 10.1007/s00382-012-1336-x

Weller, H., McIntyre, W., \& Shipley, D. (2020). Multifluids for representing subgrid-scale convection. Journal of Advances in Modeling Earth Systems, 12(8), e2019MS001966. Retrieved from https://agupubs.onlinelibrary .wiley.com/doi/abs/10.1029/2019MS001966 10.1029/2019MS001966) doi: https://doi.org/10.1029/2019MS001966

Weller, H., \& McIntyre, W. A. (2019). Numerical solution of the conditionally averaged equations for representing net mass flux due to convection. Quarterly Journal of the Royal Meteorological Society, 145(721), 1337-1353. doi: $10.1002 /$ qj. 3490

Yano, J. (2014). Basic convective element: Bubble or plume? a historical review. Atmos. Chem. Phys, 14(13), 7019-7030. doi: https://doi.org/10.5194/acp-14 $-7019-2014$ 